\title{
Unique characteristics of $\mathrm{CpG}$ island methylator phenotype (CIMP) in a Chinese population with colorectal cancer
}

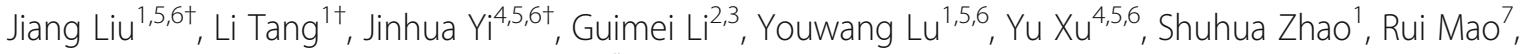
Xiaolu Li ${ }^{2,3}$, Li Ren ${ }^{8}$ and Kunhua Wang ${ }^{4,5,6^{*}}$

\begin{abstract}
Background: Molecular characteristics of CpG island methylator phenotype (CIMP) in colorectal cancer (CRC) have been well documented in Western, but not in Chinese, populations.

Methods: We investigated the incidence of CIMP, BRAF/KRAS mutation, and microsatellite instability (MSI) in a Chinese population with CRC $(n=401)$ and analysed associations between CIMP status and clinicopathological and molecular features.

Results: A total of 41 cases, 310 cases, and 40 cases were classified as CIMP-high, CIMP-low, and CIMP-negative, respectively. We detected a significantly low incidence of BRAF mutation in adenomas (2\%) and CRC (0.7\%), and a relatively low incidence of KRAS mutation (24.9\%) compared with that in other populations. We also detected a relatively low incidence of CIMP-high (10.2\%), which was significantly associated with younger age ( $\leq 49$ years of age), female sex, and proximal tumour location.

Conclusions: This study revealed unique characteristics of CIMP in a Chinese population with colorectal cancer. Developing specific CIMP markers based on unique populations or ethnic groups will further help to fully elucidate CIMP pathogenesis.
\end{abstract}

Keywords: Colorectal cancer, CpG island methylator phenotype, CIMP, BRAF mutations, KRAS mutations

\section{Background}

Based on global estimates in 2012, colorectal cancer (CRC) is the third most commonly diagnosed cancer in males and the second in females, with an estimated 1.4 million cases and 693,900 deaths occurring annually [1]. CRC is currently considered to represent a constellation of heterogeneous subtypes that result from different combinations of genetic events and epigenetic alterations. A series of studies have shown the ability to classify CRC subtypes based on combinations of microsatellite instability (MSI), CpG island methylator phenotype (CIMP),

\footnotetext{
* Correspondence: kunhuawang1@163.com

${ }^{\dagger}$ Jiang Liu, Li Tang and Jinhua Yi contributed equally to this work. ${ }^{4}$ Department of Gastrointestinal Surgery, the First Affiliated Hospital of Kunming Medical University, Yunnan Institute of Digestive Disease, Kunming 650032, Yunnan, China

${ }^{5}$ Yunnan Engineering Technology Center of Digestive Disease, Kunming 650032, Yunnan, China

Full list of author information is available at the end of the article
}

somatic BRAF mutation, and/or somatic KRAS mutation status [2-11]. Over the years, significant advances have been made in characterizing the molecular genetics and epigenetics of colorectal tumourigenesis, leading to the bench-to-bedside application of biomarkers such as KRAS, $B R A F$, and CIMP for personalised medicine [12].

CIMP characterises a subset of CRCs exhibiting a very high frequency of aberrant DNA hypermethylation at "type C" loci, which are defined as loci methylated in cancer, but not in normal, tissues [13]. The CIMP trait has been found to be associated with a variety of clinical, histopathological, and epidemiological characteristics, such as older age, female sex, proximal tumour location, poorly differentiated or mucinous histology, and high rates of MSI and BRAF mutation [4, 6, 9, 10, 14-17]. Although there are conflicting data regarding whether CIMP-positive patients receive benefit from adjuvant 5fluorouracil therapy $[4,9,18,19]$, CIMP status has been

(C) The Author(s). 2019 Open Access This article is distributed under the terms of the Creative Commons Attribution 4.0 International License (http://creativecommons.org/licenses/by/4.0/), which permits unrestricted use, distribution, and 
evaluated as a predictive marker for chemotherapy responsiveness. Possible explanations for this inconsistency include the use of small case-control studies, differences in the loci used to define CIMP, and different hypermethylation assays used.

Although accumulating evidence indicates that these molecular characteristics (including MSI, CIMP, and $B R A F / K R A S$ mutation status) have diagnostic, therapeutic, and prognostic significance in CRC [20-22], their incidences, especially those of MSI, BRAF mutation, and CIMP, vary considerably among different ethnic groups $[8,22,23]$. In a population-based cohort study, Carethers et al. found that the frequency of MSI among an AfricanAmerican cohort with colon cancer was half that of a Caucasian cohort, suggesting that once an African-American is diagnosed with colon cancer, the improved survival associated with MSI cancers is more limited in this population [24]. Worldwide, the reported frequency of BRAF mutation in different populations varies widely, from $1.1 \%$ in Taiwan to $19.8 \%$ in the Netherlands [5, 22, 25-30]. Compared with Western populations, a lower frequency of BRAF mutation has been observed in most Asian populations, from $1.1 \%$ in Taiwan to $7 \%$ in North China [5, 25, $26,28-30]$. Similarly, the reported incidence of CIMP in different populations varies widely, from $5.1 \%$ in Saudi Arabia to 30\% in the United States [2, 6-8, 10, 11, 31-35]. These differences may be related to differences in the methodology and CIMP marker panel used to determine CIMP status in these studies [33]. In addition, studies focusing on the molecular characteristics of CIMP in Chinese populations are very limited. The only relevant study reports a CIMP incidence of $13.12 \%$ in a Northeast Chinese population [7]; however, it has been suggested that the CIMP markers used in this study (including MINT1, MINT31, p16, MLH1, MGMT, APC, and RUNX3) are not very sensitive and specific for CIMP diagnosis [31].

Due to the lack of data on the molecular characteristics of CRC (including MSI, CIMP, and BRAF/KRAS mutation status) in Chinese populations, we sought to utilise a population-based CRC cohort to more accurately determine the prevalence and characteristics of these features in a Chinese CRC population. We comprehensively investigated the incidence of MSI, CIMP, and BRAF and KRAS mutations in a unique ethnic
Chinese CRC population and analysed associations between CIMP status and clinicopathological and molecular features. We also aimed to elucidate the aetiological factors and pathogenesis of CIMP-high CRC in this unique ethnic Chinese population of CRC patients.

\section{Methods}

\section{Tissue samples}

Formalin-fixed, paraffin-embedded archival tissues from 317 CRC patients were retrieved from the Department of Pathology, the First Affiliated Hospital of Kunming Medical University (Kunming, China). Fresh colorectal tumour and surrounding normal tissues were collected at surgery from 84 patients, and representative sections for research were removed by a pathologist. These patients had undergone curative surgery at the First Affiliated Hospital of Kunming Medical University between 2014 and 2016. Patients gave a written informed consent for the use of their bowel tissue for research. Selection was based solely on the availability of archival tissue blocks for the study, and we did not exclude patients with a family history of CRC. Clinicopathological information, including age, sex, tumour location, and tumour stage, was obtained from all 401 patients (Table 1). The cecum, ascending colon, hepatic flexure, transverse colon, and splenic flexure were classified as proximal, while the descending colon, sigmoid colon, and rectum were classified as distal. Tumours were staged on the basis of the pathological tumour-nodemetastasis (pTNM) staging system of the American Joint Committee on Cancer (AJCC). The study was approved by the First Affiliated Hospital of Kunming Medical University Ethics Committee.

\section{DNA extraction and bisulphite modification}

Through light microscopic examination, we marked tumour areas where tumour cells accounted for $50 \%$ or more of all cells and analysed the main histology and differentiation of the tumour. Eight serial $10-\mu \mathrm{m}$-thick histological slides of formalin-fixed tumour tissue blocks were used for manual microdissection. Genomic DNA was extracted using the QIAamp DNA FFPE Tissue kit (QIAGEN, Germantown, MD, USA) for formalin-fixed, paraffin-embedded archival tissues and the QIAamp

Table 1 Sample informations used in this study

\begin{tabular}{llllllll}
\hline & $\begin{array}{l}\text { Normal Tissues } \\
(n=84)\end{array}$ & $\begin{array}{l}\text { Adenoma } \\
(n=98)\end{array}$ & Stagel $(n=77)$ & Stagell $(n=161)$ & Stagell $(n=148)$ & StagelV $(n=15)$ & Total $(\mathrm{n}=401)$ \\
\hline Male, $\mathrm{n}(\%)$ & $48(57 \%)$ & $58(59 \%)$ & $40(52 \%)$ & $93(58 \%)$ & $85(57 \%)$ & $10(67 \%)$ & $228(57 \%)$ \\
Female, $\mathrm{n}(\%)$ & $36(43 \%)$ & $40(41 \%)$ & $37(48 \%)$ & $68(42 \%)$ & $63(43 \%)$ & $5(33 \%)$ & $173(43 \%)$ \\
Age, median (range) & $57(24-81)$ & $59(20-86)$ & $55.18(24-81)$ & $54.13(28-80)$ & $53.91(20-87)$ & $64.4(26-85)$ & $54.64(20-87)$ \\
Colon, n (\%) & $42(50 \%)$ & $69(70 \%)$ & $31(40 \%)$ & $95(59 \%)$ & $76(51 \%)$ & $12(80 \%)$ & $214(53 \%)$ \\
Rectum, $\mathrm{n}(\%)$ & $42(50 \%)$ & $29(30 \%)$ & $46(60 \%)$ & $66(41 \%)$ & $72(49 \%)$ & $3(20 \%)$ & $187(47 \%)$ \\
\hline
\end{tabular}


DNA Mini Kit (QIAGEN) for fresh tissues. Bisulphite modification was carried out using an EpiTect Fast DNA Bisulphite Kit (QIAGEN) according to the manufacturer's instructions.

\section{DNA methylation analysis}

DNA methylation analyses were performed using MethyLight, as previously described [31, 32]. The oligonucleotide sequences of the primers and probes have been described previously $[31,32,36]$. The PCR conditions were as follows: initial denaturation at $95^{\circ} \mathrm{C}$ for $10 \mathrm{~min}$, followed by 40 cycles of $95^{\circ} \mathrm{C}$ for $15 \mathrm{~s}$ and $60^{\circ} \mathrm{C}$ for $1 \mathrm{~min}$. M.SssI-treated genomic DNA was used as a completely methylated reference sample to determine the percentage of fully methylated alleles [percentage of methylated reference (PMR)] at a particular locus. The PMR value was calculated by dividing the GENE/ALU ratio of a sample by the GENE/ALU ratio of the M.SssItreated human genomic DNA sample and multiplying by 100. A PMR cut-off of 4 was used to distinguish methylation-positive $(\mathrm{PMR}>4)$ from methylation-negative $(\mathrm{PMR} \leq 4)$ samples.

Although several marker panels have been proposed to standardise the classification of CIMP-positive [31, 32, 36, 37], we quantified DNA methylation in eight CIMP markers (CACNA1G, CDKN2A, CRABP1, IGF2, MLH1, NEUROG1, RUNX3, and SOCS1), as these have been shown to be sensitive and specific for CIMP diagnosis [32]. These eight CIMP markers can be divided into three marker panels: CIMP-1 (CACNA1G, CDKN2A, CRABP1, MLH1, and NEUROG1), described by Ogino and colleagues [36]; CIMP-2 (CACN A1G, IGF2, NEUROG1, RUNX3, and SOCS1), described by Weisenberger and colleagues [31]; and CIMP-3, including all eight of the markers [32, 33, 38]. For the CIMP-1 panel, CRC cases were considered CIMP-positive if at least four of the markers were methylated [36]; for the CIMP-2 panel, CRC cases were considered CIMP-positive if at least three of the markers were methylated [31]. For the CIMP-3 panel, a cut-off of $\geq 5 / 8$ methylated markers was used to classify cases into CIMP-high CRC, as usage of this cut-off has shown stronger associations with known clinicopathological or molecular features of CIMP-high CRC in Korea [33]. A cut-off of 1-4/8 methylated markers was used to classify cases into CIMP-low, while a cut-off of 0/8 methylated markers was used to classify cases into CIMP-negative. We evaluated the performance of these three marker panels by comparing their associations with clinicopathological features of CRC that have been previously reported to be associated with CIMP-positive status, including older age, female sex, proximal location, BRAF mutation, and MSI status.

\section{Mutational analysis of KRAS codons 12 and 13 and BRAF codon 600}

Tumour DNA was tested for the BRAF codon 597 and 600 mutations and KRAS codons 12 and 13 mutations in
98 adenomas and $401 \mathrm{CRC}$ samples. Mutations of BRAF (nucleotides 1790 and 1799) and KRAS (nucleotides 35 and 38) were analysed by genotyping assay on the MassARRAY platform (Sequenom, San Diego, CA, USA). PCR and extension primers for these mutations were designed using MassARRAY Assay Design 3.0 software (Sequenom) and applying default single-base extension settings and default parameters (Additional file 1: Table S1). DNA was amplified by PCR, and a single-base extension reaction was performed using a custom mixture of nucleotides and extension primers that hybridised immediately adjacent to the mutations. Reaction products were transferred to a SpectroCHIP (Sequenom), and mass differences were analysed using MALDI-TOF mass spectrometry to identify the extended base at the possible mutation site. Repeat Sanger sequencing on an ABI PRISM 3730 Genetic Analyzer (Applied Biosystems, Foster City, CA, USA) was used to reconfirm the results of MassARRAY and rule out the possibility that any mutations were missed due to the sensitivity of the MassARRAY platform. Primers used to amplify and sequence exon 15 of BRAF and exon 1 of KRAS are shown in Additional file 1: Table S1.

\section{MSI analysis}

For determination of MSI status, we used a panel of 5 microsatellite markers (BAT25, BAT26, NR-21, NR-24, and $M O N O-27)$ to classify fresh tumour tissues as MSIhigh (MSI-H), MSI-low (MSI-L), or microsatellite stable (MSS). MSI-H was defined as $\geq 2$ markers demonstrating novel alleles compared to non-tumour tissues, MSI-L was defined as 1 marker with a novel allele, and MSS was defined as no markers with novel alleles.

\section{Statistical analysis}

For statistical analysis, the $\chi^{2}$ test or Fisher's exact test (for categories with $n<10$ ) was performed on categorical data using the IBM SPSS Statistics 22.0 software. All $P$ values were two-sided, and statistical significance was set at $P \leq$ 0.05 .

\section{Results}

\section{Clinicopathological characteristics}

Of the 401 patients, the proportion of males (57\%) was slightly higher than that of females (43\%), with a male to female ratio of 1.32:1. Patient age at presentation ranged from 20 to 87 years (median, 54.64 years). There were 309 patients (77.1\%) that presented with stage II or III disease, while 77 patients (19.2\%) were diagnosed with stage I disease and 15 patients $(3.7 \%)$ were diagnosed with stage IV disease. There were 214 patients $(53.4 \%)$ whose primary tumours were derived from the colon, while the tumours in the remaining 187 patients (46.6\%) were derived from the rectum. Detailed sample information is summarised in Table 1. 


\section{$B R A F$ and KRAS mutations}

Of the 401 CRC specimens analysed for BRAF and KRAS mutations using the MassARRAY platform, BRAF mutation was observed in three cases, with an incidence of $0.7 \%$ (3/401); KRAS mutation was detected in 100 cases, with an incidence of $24.9 \%(100 / 401)$ (Table 2). For the 98 adenoma samples, BRAF mutation was observed in two cases, with an incidence of $2 \%(2 / 98)$, and KRAS mutation was detected in 23 cases, with an incidence of $23.5 \%$ (23/98). All five BRAF mutations were V600E mutations, while KRAS-mutated cases showed mutations at codon 12 (67\%) and codon 13 (33\%). Repeat Sanger sequencing was conducted for 229 specimens, including all of the BRAF- and KRAS-mutated cases. The results of repeat Sanger sequencing were in accordance with MassARRAY analyses, with no new mutations identified.

\section{MSI analysis}

MSI status was determined in 82 CRC patients due to inadequate DNA, lack of paired normal tissues, or technical issues with the remaining specimens. The incidences of MSI-H, MSI-L, and MSS were 6.1\% (5/82), $23.2 \%$ (19/82), and 70.7\% (58/82), respectively (Table 2).

\section{CIMP prevalence and correlations with clinicopathological and molecular characteristics}

We obtained 401 colorectal cancer specimens and successfully quantified DNA methylation in eight CIMPspecific gene promoters (CACNA1G, CDKN2A, CRABP1, IGF2, MLH1, NEUROG1, RUNX3, and SOCS1) using MethyLight technology. Methylation frequencies were $20 \%$ for CACNA1G (80 cases), $57.6 \%$ for CDKN2A (231 cases), $49.6 \%$ for CRABP1 (199 cases), 30.7\% for IGF2 (123 cases), 3.7\% for MLH1 (15 cases), 31.2\% for NEUROG1 (125 cases), 7.2\% for RUNX3 (29 cases), and $17 \%$ for SOCS1 (68 cases). A summary of the clinicopathological and molecular characteristics of CRC cases according to each of the three CIMP panels (CIMP-1, CIMP-2, and CIMP-3) is provided in Fig. 1 and Table 2. CIMP-positive cancers were identified in 34 cases $(8.5 \%)$

Table 2 Clinicopathologic and molecular characteristics of CRCs

\begin{tabular}{|c|c|c|c|c|c|c|c|c|c|c|}
\hline \multirow[t]{2}{*}{ Demographics } & \multicolumn{3}{|l|}{ CIMP-1 $(\geq 4 / 5)$} & \multicolumn{3}{|l|}{ CIMP-2 ( $(\geq 3 / 5)$} & \multicolumn{4}{|l|}{ CIMP-3 $(\geq 5 / 8)$} \\
\hline & Positive (8.5\%) & Negative & $P$ & Positive (12\%) & Negative & $P$ & High (10.2\%) & Low (77.3\%) & Negative (12.5\%) & $P$ \\
\hline Age & & & 0.1871 & & & 0.1108 & & & & 0.0296 \\
\hline$\leq 49$ & 15 (3.7\%) & 108 (26.9\%) & & $22(5.5 \%)$ & $101(25.2 \%)$ & & $20(5 \%)$ & 86 (21.4\%) & 17 (4.2\%) & \\
\hline $50-59$ & $11(2.7 \%)$ & 135 (33.7\%) & & $14(3.5 \%)$ & 132 (32.9\%) & & $11(2.7 \%)$ & $120(29.9 \%)$ & 15 (3.7\%) & \\
\hline$\geq 60$ & $8(2 \%)$ & $124(30.9 \%)$ & & $12(3 \%)$ & 120 (29.9\%) & & $10(2.5 \%)$ & 104 (25.9\%) & $18(4.5 \%)$ & \\
\hline Gender & & & 0.0046 & & & 0.0063 & & & & 0.0011 \\
\hline Men & $11(2.7 \%)$ & 217 (54.1\%) & & $18(4.5 \%)$ & 210 (52.4\%) & & $13(3.2 \%)$ & $186(46.4 \%)$ & $29(7.2 \%)$ & \\
\hline Women & $23(5.7 \%)$ & $150(37.4 \%)$ & & $30(7.5 \%)$ & $143(35.7 \%)$ & & $28(7 \%)$ & $124(30.9 \%)$ & $21(5.2 \%)$ & \\
\hline Tumor location & & & 0.0142 & & & 0.0552 & & & & 0.0065 \\
\hline Distal & $19(4.7 \%)$ & $281(70.1 \%)$ & & $30(7.5 \%)$ & $270(67.3 \%)$ & & $23(5.7 \%)$ & $244(60.8 \%)$ & 33 (8.2\%) & \\
\hline Proximal & $15(3.7 \%)$ & $86(21.4 \%)$ & & $18(4.5 \%)$ & $83(20.7 \%)$ & & $18(4.5 \%)$ & $66(16.5 \%)$ & $17(4.2 \%)$ & \\
\hline Stage & & & 0.1697 & & & 0.5059 & & & & 0.5459 \\
\hline । & $5(1.2 \%)$ & 72 (18.0\%) & & $9(2.2 \%)$ & $68(17 \%)$ & & $6(1.5 \%)$ & 61 (15.2\%) & $10(2.5 \%)$ & \\
\hline ॥ & 17 (4.2\%) & $144(35.9 \%)$ & & $24(6 \%)$ & $137(34.2 \%)$ & & $21(5.2 \%)$ & 119 (29.7\%) & $21(5.2 \%)$ & \\
\hline III & $9(2.2 \%)$ & $139(34.7 \%)$ & & $14(3.5 \%)$ & 134 (33.4\%) & & $13(3.2 \%)$ & $120(29.9 \%)$ & 15 (3.7\%) & \\
\hline IV & $3(0.7 \%)$ & $12(3.0 \%)$ & & $1(0.2 \%)$ & 14 (3.5\%) & & $1(0.2 \%)$ & $10(2.5 \%)$ & $4(1 \%)$ & \\
\hline BRAF status & & & 0.2339 & & & 0.039 & & & & 0.2771 \\
\hline Wild type & 33 (8.2\%) & 365 (91.0\%) & & 46 (11.5\%) & 352 (87.8\%) & & 40 (10\%) & 308 (76.8\%) & $50(12.5 \%)$ & \\
\hline Mutation & $1(0.2 \%)$ & $2(0.5 \%)$ & & $2(0.5 \%)$ & $1(0.2 \%)$ & & $1(0.2 \%)$ & $2(0.5 \%)$ & $0(0)$ & \\
\hline KRAS status & & & 0.0957 & & & 0.1072 & & & & 0.1033 \\
\hline Wild type & $21(5.2 \%)$ & $280(69.8 \%)$ & & $31(7.7 \%)$ & 270 (67.3\%) & & $26(6.5 \%)$ & 234 (58.4\%) & 41 (10.2\%) & \\
\hline Mutation & $13(3.2 \%)$ & 87 (21.7\%) & & 17 (4.2\%) & 83 (20.7\%) & & 15 (3.7\%) & 76 (19\%) & $9(2.2 \%)$ & \\
\hline MSI status & & & 1 & & & 0.287 & & & & 0.7373 \\
\hline MSS & $5(6.1 \%)$ & $53(64.6 \%)$ & & 10 (12.2\%) & 48 (58.5\%) & & 8 (9.8\%) & 44 (53.7\%) & $6(7.3 \%)$ & \\
\hline MSI-low & $1(1.2 \%)$ & 18 (22.0\%) & & $2(2.4 \%)$ & 17 (20.7\%) & & $2(2.4 \%)$ & 15 (18.3\%) & $2(2.4 \%)$ & \\
\hline MSI-high & $0(0)$ & $5(6.1 \%)$ & & $2(2.4 \%)$ & $3(3.7 \%)$ & & $1(1.2 \%)$ & $4(4.9 \%)$ & $0(0)$ & \\
\hline
\end{tabular}




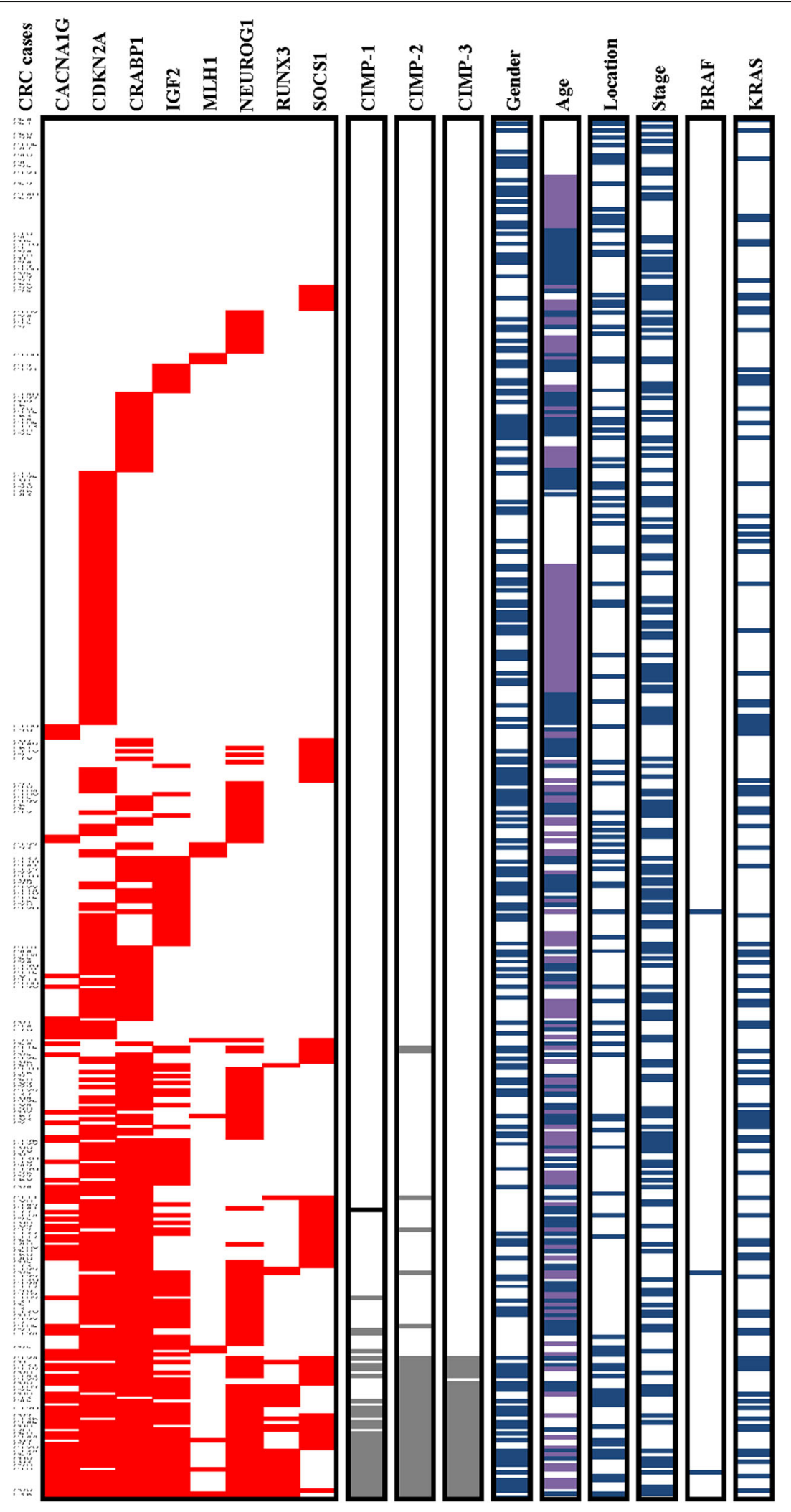

Fig. 1 Comparative analysis of CIMP marker panel performance. Red bars represent methylation-positive CIMP markers, and grey bars represent CIMP-positive (CIMP-1 and CIMP-2) or CIMP-high (CIMP-3) classifications using three differently defined CIMP panels. Blue bars represent female sex, older age ( $\geq 60$ years), proximal colon location, higher stage (III, IV), KRAS mutation, and BRAF mutation. Purple bars represent ages 50-59 
using the CIMP-1 panel and 48 cases (12\%) using the CIMP-2 panel. For the CIMP-3 marker panel, 41 cases (10.2\%), 310 cases $(77.3 \%)$, and 50 cases (12.5\%) were classified as CIMP-high, CIMP-low, and CIMP-negative, respectively. The frequency of CIMP-1-positive cases was significantly higher in women (13.3\%) than in men $(4.8 \%, P<0.01)$ and significantly higher in cases with proximal tumour locations $(14.9 \%)$ than in those with distal tumour locations $(6.3 \%, P<0.05)$. CIMP-2 positivity was significantly more frequent in women $(17.3 \%)$ than in men $(7.9 \%, P<0.001)$ and was associated with a $B R A F$ mutant type (66.7\%) rather than $B R A F$ wild type (11.6\%, $P<0.05)$. For the CIMP-3 panel, of particular note is the fact that the frequency of CIMP-high varied by age, with a significantly higher rate in patients $\leq 49$ years of age (16.3\%) compared to that in patients $50-59$ years of age $(7.5 \%)$ and $\geq 60$ years of age $(7.6 \%, P<0.05)$. Moreover, CIMP-high was significantly more frequent in women $(16.2 \%)$ than in men $(5.7 \%, P<0.01)$ and in proximal tumour locations (17.8\%) than in distal tumour locations $(7.7 \%, P<0.01)$. No significant differences were observed in other clinicopathological characteristics among the CIMP phenotypes for the three panels of CIMP markers. Because previous studies suggested that the CIMP-3 panel outperformed the CIMP-1 and CIMP-2 panels both in Western CRC populations and in Asian CRC populations [32, 33], we used the CIMP-3 panel for the determination of CIMP in this study.

\section{Assessment of individual CIMP methylation markers}

To compare the performance of the eight individual methylation markers for the determination of panelspecific CIMP status, the sensitivity and specificity were calculated for each of the eight markers among all 401 tumours (Table 3). As shown in Table 3, CACNA1G, $C D K N 2 A, C R A B P 1$, and NEUROG1 demonstrated very high sensitivity $(\geq 97 \%)$ in determining CIMP-1 status. Similarly, CRABP1 and NEUROG1 demonstrated high sensitivity $(\geq 90 \%)$ in determining CIMP-2 status, and CDKN2A, CRABP1, and NEUROG1 demonstrated very high sensitivity $(\geq 97 \%)$ in determining CIMP-3 status. For all three panels, MLH1 and RUNX3 exhibited superior specificity $(\geq 96 \%)$.

\section{Discussion}

Due to accumulating evidence indicating that certain molecular characteristics (including CIMP, BRAF mutation, KRAS mutation, and MSI status) have diagnostic, therapeutic, and prognostic significance in CRC personalised medicine and incidences that vary considerably among different ethnic or geographic populations, this study determined the frequency of CIMP, BRAF/KRAS mutation, and MSI in a unique ethnic Chinese population-based CRC cohort. Surprisingly, we detected a significantly low incidence of $B R A F$ mutation, both in adenomas $(2 \%)$ and in CRC (0.7\%), and a relatively low incidence of KRAS mutation (24.9\%) compared with that in other populations $[9,10,29,32,33,39,40]$. We also detected a relatively low incidence of CIMP-high (10.2\%) in our CRC population. Of note, CIMP-high was significantly associated with younger age ( $\leq 49$ years old), female sex, and proximal tumour location, whereas no significant associations were observed with tumour stage, $B R A F$ mutation, KRAS mutation, or MSI status. In addition, by comparing the accuracy of the associations of the three CIMP marker panels with previously known clinicopathological features of CIMP-positive CRC, our data indicated that the CIMP-3 panel outperformed the CIMP-1 and CIMP-2 panels in most comparisons. Therefore, consistent with analyses in American and South Korean CRC populations [32, 33], CIMP-3 is currently the optimal marker panel for the determination of CIMP status in the Chinese population with CRC.

$B R A F$ mutation has been considered a biomarker with diagnostic, therapeutic, and prognostic significance in CRC $[41,42]$. In this study, the BRAF-V600E mutation was identified in only $0.7 \%(3 / 401)$ of all CRC cases and $2 \%(2 / 98)$ of adenoma cases. This implies a very limited role of the $B R A F$ gene in the pathogenic process of CRC and a much lower clinical significance of $B R A F$ mutation in Chinese populations than in Western populations [2, $6,9,10] . B R A F$ mutation is tightly associated with MSI$\mathrm{H}$ and MLH1 methylation in Western CRC populations $[31,42]$, but the main reason of MSI-H $(6.1 \%, 5 / 82)$ and MLH1 methylation $(3.7 \%, 15 / 401)$ in Chinese CRC populations remains unclear due to very low incidences of $B R A F$ mutation. Although very low incidences of $B R A F$ mutation were observed in Saudi Arabia and Israel, with frequencies of 2.5 and $5 \%$, respectively [8,43], and lower frequencies have been observed in most Asian populations, ranging from $1.1 \%$ in Taiwan [28] to $2.3-7 \%$ in China [25, 29, 44], 4.7-6.7\% in Japan [5, 30], and 4.1\% in South Korea [40], the incidence revealed in this study is the lowest observed thus far compared with previous reports worldwide $[8,10,42]$. In addition, concordant with previous reports that the incidence of $B R A F$ mutation varies widely among CRC populations even within the same region or country $[8,45,46]$, three previous studies have reported varied incidences of $B R A F$ mutation in CRC populations from different areas of China. A $B R A F$ mutation frequency of $2.3 \%(5 / 220)$ was observed in Shanghai [44], 4.4\% (20/453) was observed in Beijing [29], and 7\% (14/200) was observed in Shanxi province [25], with the lowest incidence of $0.7 \%$ (3/401) from the population in this study from Yunnan province. Yunnan province has the most ethnic minorities in China: among the 26 nationalities in Yunnan, 15 of them are native ethnic minorities. Therefore, the fact that the lowest 
Table 3 Sensitivity and specificity of each marker for determination of CIMP-high

\begin{tabular}{|c|c|c|c|c|c|c|c|}
\hline \multirow[t]{2}{*}{ Marker } & \multirow[t]{2}{*}{ Total no. } & \multicolumn{2}{|c|}{ CIMP-1 ( $(\geq 4 / 5)$} & \multicolumn{2}{|l|}{ CIMP-2 ( $\geq 3 / 5)$} & \multicolumn{2}{|l|}{ CIMP-3 ( $\geq 5 / 8)$} \\
\hline & & $\begin{array}{l}\text { Positive } \\
\text { (sensitivity) }^{\text {a }}\end{array}$ & 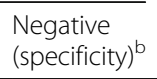 & $\begin{array}{l}\text { Positive } \\
\text { (sensitivity) a }^{\text {a }}\end{array}$ & $\begin{array}{l}\text { Negative } \\
\text { (specificity) }^{\text {b }}\end{array}$ & $\begin{array}{l}\text { Positive } \\
\text { (sensitivity) }{ }^{\text {a }}\end{array}$ & $\begin{array}{l}\text { Negative } \\
\text { (specificity) }^{\text {b }}\end{array}$ \\
\hline & 401 & $34(8.5 \%)$ & 367 & $48(12 \%)$ & 353 & 41 (10.2\%) & 360 \\
\hline \multicolumn{8}{|c|}{ CACNAIG } \\
\hline$(+)$ & $80(20 \%)$ & $33(97 \%)$ & 47 & $38(79 \%)$ & 42 & 34 (83\%) & 46 \\
\hline$(-)$ & 321 & 1 & $320(87 \%)$ & 10 & 311 (88\%) & 7 & 314 (87\%) \\
\hline \multicolumn{8}{|c|}{ CDKN2A } \\
\hline$(+)$ & $231(57.6 \%)$ & 33 (97\%) & 198 & 38 (79\%) & 193 & 37 (90\%) & 194 \\
\hline$(-)$ & 170 & 1 & 169 (46\%) & 10 & $160(45 \%)$ & 4 & $166(46 \%)$ \\
\hline \multicolumn{8}{|l|}{ CRABP1 } \\
\hline$(+)$ & 199 (49.6\%) & $34(100 \%)$ & 165 & 45 (94\%) & 154 & 40 (98\%) & 159 \\
\hline$(-)$ & 202 & 0 & 202 (55\%) & 3 & 199 (56\%) & 1 & 201 (56\%) \\
\hline \multicolumn{8}{|l|}{ IGF2 } \\
\hline$(+)$ & $123(30.7 \%)$ & $24(71 \%)$ & 99 & 40 (83\%) & 83 & 34 (83\%) & 89 \\
\hline$(-)$ & 278 & 10 & $268(73 \%)$ & 8 & $270(77 \%)$ & 7 & 271 (75\%) \\
\hline \multicolumn{8}{|l|}{ MLH1 } \\
\hline$(+)$ & 15 (3.7\%) & $5(15 \%)$ & 10 & $4(8.3 \%)$ & 11 & $4(9.8 \%)$ & 11 \\
\hline$(-)$ & 386 & 29 & 357 (97\%) & 44 & 342 (97\%) & 37 & 349 (97\%) \\
\hline \multicolumn{8}{|c|}{ NEUROG1 } \\
\hline$(+)$ & $125(31.2 \%)$ & $33(97 \%)$ & 92 & $43(90 \%)$ & 82 & 39 (95\%) & 86 \\
\hline$(-)$ & 276 & 1 & 275 (74\%) & 5 & 271 (77\%) & 2 & $274(76 \%)$ \\
\hline \multicolumn{8}{|l|}{ RUNX3 } \\
\hline$(+)$ & $29(7.2 \%)$ & $16(47 \%)$ & 13 & 27 (56\%) & 2 & $25(61 \%)$ & 4 \\
\hline$(-)$ & 372 & 18 & 354 (96\%) & 21 & 351 (99\%) & 16 & 356 (99\%) \\
\hline \multicolumn{8}{|l|}{ SOCS1 } \\
\hline$(+)$ & 68 (17\%) & 13 (38\%) & 55 & $24(50 \%)$ & 44 & 19 (46\%) & 49 \\
\hline$(-)$ & 333 & 21 & 312 (85\%) & 24 & 309 (88\%) & 22 & 311 (86\%) \\
\hline
\end{tabular}

${ }^{a}$ Sensitivity of each marker is defined as the number of CIMP-high cases positive for a given marker divided by the number of all CIMP-high cases

${ }^{b}$ Specificity of each marker is defined as the number of non-CIMP-high cases negative for a given marker divided by the number of all non-CIMP-high cases

incidence of $B R A F$ mutation was observed in a Yunnan CRC population may be due to differences in ethnic populations and the associated variation in underlying genetic and epigenetic backgrounds, as well as environmental influences such as food habits, smoking, drinking, and other unknown factors.

The CIMP-1 panel was first developed by Ogino and colleagues [36]; in their study, 17\% (78) of the 460 evaluated CRC specimens were classified as CIMP-positive. In our study, CIMP-1-positive cancers were identified in 34 cases (8.5\%) among the 401 CRC specimens. CIMP-2 was first developed by Weisenberger and colleagues [31], who reported that $18 \%$ (33) of their 187 CRC specimens were classified as CIMP-positive. In our study, CIMP-2positive cancers were identified in 48 cases $(12 \%)$ of the 401 CRC specimens. In 2007, the CIMP-3 panel was first proposed by Ogino and colleagues [32]. In their study, $18 \%$ (163) of the 920 CRC specimens were classified as
CIMP-high. Later, Kim et al. used the same CIMP marker panel and classified 12\% (37/320) of South Korean CRC patients as CIMP-high [33]. In our study, CIMP-high cancers were identified in 41 cases $(10.2 \%)$ of the 401 CRC specimens. In general, based on the same CIMP marker panel and the same CIMP-high criterion, the frequency of CIMP-high cancers in our CRC population was relatively lower than that of the American CRC population but similar to that of the South Korean population. Of note, although two previous studies determined the frequency of CIMP-positive cancers in Chinese CRC populations [7, 47], these did not use the recognised CIMP markers that we used in this study to classify CIMP cancers, so their results are not comparable with ours and others'. Li et al. used MLH1, MGMT, p16, APC, MINT1, MINT31, and RUNX3 as CIMP panel markers and classified $13.12 \%(37 / 282)$ of patients as CIMP-high [7]. Wang et al. used p14ARF, hMLH1, 
p16INK4a, MGMT, and MINT1 as CIMP markers and identified 12 CIMP-positive cases (24\%) in 50 CRC specimens [47]. Therefore, our results once again show that differences in CIMP marker panels may contribute to discrepancies in CIMP frequency, even for the same CRC population.

Studies on American CRC populations have indicated that CIMP is significantly associated with female sex, older age, proximal tumour location, MSI, BRAF mutation, and wild-type KRAS [31, 36, 48]. However, in this study, CIMP-high was significantly associated with female sex, younger age, and proximal tumour location. No significant association was observed with other clinicopathological characteristics, including MSI, BRAF mutation, or wild-type KRAS. Notably, this is the first study to report an association between CIMP-high and younger age. A possible reason for the lack of an association between CIMP-high and MSI may be the limited sample size $(n=82)$ included in the MSI analysis. The lack of an association with $B R A F$ mutation may be due to the very low incidence of BRAF mutation observed in this population $(0.7 \%)$. Alternatively, just as accumulating evidence has demonstrated that differences in CIMP marker panels may contribute to discrepancies in CIMP frequency, even in the same CRC population, the same CIMP marker panel may not be suitable for the diagnosis of CIMP among different populations. For example, using the same CIMP-3 marker panel with the same CIMP-high criterion, Ogino and colleagues found that CRABP1, IGF2, and NEUROG1 demonstrated very good sensitivity ( $\geq 95 \%)$, whereas $C A C N$ A1G, MLH1, RUNX3, and SOCS1 showed superior specificity $(\geq 90 \%)$ in 920 American CRC cases [32]. However, in this study, we found that $C D K N 2 A$, CRABP1, and NEUROG1 demonstrated very good sensitivity ( $\geq 97 \%$ ), while $M L H 1$ and $R U N X 3$ exhibited superior specificity $(\geq 96 \%)$ when using CIMP-3. In contrast, the sensitivity of IGF2 was $83 \%$, and the specificities of CACNA1G and SOCS1 were 87 and $86 \%$, respectively, in our CRC population, while the sensitivity of CDKN2A was $87 \%$ among American CRCs [32]. Thus, the same CIMP markers exhibit different performances for the determination of CIMP in different CRC populations. While all of the eight CIMP markers in the CIMP-3 marker panel were developed based on American CRC populations [31, 32, 36], increasing evidence has shown that the incidence of CIMP varies widely among different populations [41] or ethnic backgrounds [48]. Therefore, as with the low BRAF mutation frequency detected in this study, we speculate that different ethnic populations with different underlying genetic and epigenetic backgrounds and environmental influences, such as food habits, lifestyle habits, and environmental exposures, may contribute to the varied CIMP characteristics and prevalence observed. The development of specific CIMP markers based on unique CRC populations or ethnicities will further help to fully elucidate the pathogenesis of CIMP.

CIMP-positive tumours are generally thought to develop through the serrated neoplasia pathway and are associated with $B R A F$ mutation [31, 49]. Furthermore, the frequency of $B R A F$ mutation is much higher in serrated adenomas than in conventional adenomas. For example, the frequency of $B R A F$ mutation was found to be $67 \%$ among 200 traditional serrated adenomas, but no $B R A F$ mutations were identified in 50 control tubulovillous adenomas [50]. However, in this study, although the incidence of CIMP-high was $10.2 \%, B R A F$ mutation was very rare among $C R C$ cases (only $0.7 \%$ ). Similarly, the $B R A F$ mutation frequency was only $2 \%$ in adenomas. Therefore, as the acknowledged precursors of CIMPpositive CRC, the contributions of advanced serrated adenomas to the incidence of CIMP-high CRC should be very limited in our CRC population. We believe that the unique ethnic population and the associated underlying genetic and epigenetic backgrounds may contribute to the unique molecular characteristics of CIMP-high CRC in our population. Associations between CIMP-positive CRC and environmental exposures have been carefully investigated, and associations of smoking and obesity with CIMP-positive CRC were evident only for females in an American population [48]. Therefore, characterising CIMP-high CRC using genome-scale technologies and dissecting the separate aetiological factors associated with smoking, alcohol use, obesity, and physical inactivity will further elucidate the pathogenesis of CIMP-high CRC for this unique ethnic population.

\section{Conclusions}

This study detected a significantly low incidence of BRAF mutation in adenomas (2\%) and CRC $(0.7 \%)$, and a relatively low incidence of KRAS mutation (24.9\%) compared with that in other populations. We also detected a relatively low incidence of CIMP-high (10.2\%), which was significantly associated with younger age ( $\leq 49$ years of age), female sex, and proximal tumour location. To our knowledge, this is the first study to suggest an association between CIMP-high and younger age, while most previous studies have associated CIMPhigh and older age. We speculate that different genetic backgrounds and lifestyle habits may contribute to the unique pathogenesis of CIMP-high CRC among the ethnic Chinese population. Developing specific CIMP markers based on unique populations or ethnic groups will further help to fully elucidate CIMP pathogenesis. 


\section{Supplementary information}

Supplementary information accompanies this paper at https://doi.org/10. 1186/s12876-019-1086-x.

Additional file 1: Table S1. KRAS and BRAF primers used for MassARRAY analyses and Sanger sequencing in this study.

\section{Abbreviations}

AJCC: American Joint Committee on Cancer; CIMP: CpG island methylator phenotype; CRC: Colorectal cancer; MSI: Microsatellite instability; MSI-H: MSI high; MSI-L: MSI-low; MSS: Microsatellite stable; PMR: Percentage of methylated reference; pTNM: Pathological tumour-node-metastasis

\section{Acknowledgements}

We thank professors Guoqing Pan and Qiong Nan for their assistance in sample collection.

\section{Authors' contributions}

$J L, L T$, and KHW designed the study. $J$, JHY, GML and XLL carried out the experiment work. JL, YWL, and RM performed the data analyses. $J \mathrm{~L}, \mathrm{LT}$, and KHW wrote the manuscript. HYL contributed to the manuscript revision. YX SHZ and LR helped collect samples. All authors read and approved the final manuscript.

\section{Funding}

This work was supported by grants from Yunnan Applied Basic Research Projects-Union Foundation of Kunming Medical University [2017FE467(132)], the National Natural Science Foundation of China (81960504 and 3171101074), Yunling Scholar (YLXL20170002), Key Science and Technology Planning Project of Kunming Science and Technology Bureau, Reproductive and Genetic Technology Innovation Team of Kunming Medical University (CXTD201708), the Hundred-Talent Program of Kunming Medical University (60118260104) and Doctoral Research Intial Funding of the First Affiliated Hospital of Kunming Medical University (2017BS003). The funding body did not participate in the design of the study and collection, analysis, and interpretation of data and in writing the manuscript.

\section{Availability of data and materials}

The datasets generated and analyzed during the current study are available from the corresponding author on reasonable request.

\section{Ethics approval and consent to participate}

This study was conducted in accordance with the Declaration of Helsinki. The protocol was approved by the Ethics Committee of the First Affiliated Hospital of Kunming Medical University. Patients gave informed consent in writing to the use of their bowel tissue for research.

\section{Consent for publication}

Not Applicable.

\section{Competing interests}

The authors declare that they have no competing interests.

\section{Author details}

'Department of Reproduction and Genetics, the First Affiliated Hospital of Kunming Medical University, Kunming 650032, Yunnan, China. ${ }^{2}$ Public Technical Service Center, Kunming Institute of Zoology, Chinese Academy of Sciences, Kunming 650032, Yunnan, China. ${ }^{3}$ Kunming Biological Diversity Regional Center of Large Apparatus and Equipments, Chinese Academy of Sciences, Kunming 650032, Yunnan, China. ${ }^{4}$ Department of Gastrointestinal Surgery, the First Affiliated Hospital of Kunming Medical University, Yunnan Institute of Digestive Disease, Kunming 650032, Yunnan, China. ${ }^{5}$ Yunnan Engineering Technology Center of Digestive Disease, Kunming 650032, Yunnan, China. ${ }^{6}$ Kunming Engineering Technology Center of Digestive Disease, Kunming 650032, Yunnan, China. ${ }^{7}$ School of Stomatology, Kunming Medical University, Kunming 650500, Yunnan, China. ${ }^{8}$ Department of Reproductive Gynecology, the First People's Hospital of Yunnan Province, Kunming 650031, Yunnan, China.
Received: 28 March 2019 Accepted: 2 October 2019

Published online: 05 November 2019

\section{References}

1. Torre LA, Bray F, Siegel RL, Ferlay J, Lortet-Tieulent J, Jemal A. Global cancer statistics, 2012. CA-Cancer J Clin. 2015;65(2):87-108.

2. Hughes LA, Simons CC, van den Brandt PA, Goldbohm RA, de Goeij AF, de Bruine AP, van Engeland M, Weijenberg MP. Body size, physical activity and risk of colorectal cancer with or without the $\mathrm{CpG}$ island methylator phenotype (CIMP). PLoS One. 2011;6(4):e18571.

3. Yano $Y$, Konishi $K$, Yamochi T, Katagiri A, Nozawa H, Suzuki H, Toyota M, Kubota Y, Muramoto T, Kobayashi Y, et al. Clinicopathological and molecular features of colorectal serrated neoplasias with different mucosal crypt patterns. Am J Gastroenterol. 2011;106(7):1351-8.

4. Jover R, Nguyen TP, Perez-Carbonell L, Zapater P, Paya A, Alenda C, Rojas E, Cubiella J, Balaguer F, Morillas JD, et al. 5-fluorouracil adjuvant chemotherapy does not increase survival in patients with CpG island methylator phenotype colorectal cancer. Gastroenterology. 2011;140(4): 1174-81.

5. Nakanishi R, Harada J, Tuul M, Zhao Y, Ando K, Saeki H, Oki E, Ohga T, Kitao $\mathrm{H}$, Kakeji $\mathrm{Y}$, et al. Prognostic relevance of KRAS and BRAF mutations in Japanese patients with colorectal cancer. Int J Clin Oncol. 2013:18(6):1042-8.

6. Samadder NJ, Vierkant RA, Tillmans LS, Wang AH, Weisenberger DJ, Laird PW, Lynch CF, Anderson KE, French AJ, Haile RW, et al. Associations between colorectal cancer molecular markers and pathways with clinicopathologic features in older women. Gastroenterology. 2013;145(2): $348-56$

7. Li X, Hu F, Wang Y, Yao X, Zhang Z, Wang F, Sun G, Cui BB, Dong X, Zhao Y. $\mathrm{CpG}$ island methylator phenotype and prognosis of colorectal cancer in Northeast China. Biomed Res Int. 2014;2014:236361.

8. Siraj AK, Bu R, Prabhakaran S, Bavi P, Beg S, Al Hazmi M, Al-Rasheed M Alobaisi $\mathrm{K}$, Al-Dayel $\mathrm{F}$, AlManea $\mathrm{H}$, et al. A very low incidence of BRAF mutations in middle eastern colorectal carcinoma. Mol Cancer. 2014;13:168.

9. Shiovitz S, Bertagnolli MM, Renfro LA, Nam E, Foster NR, Dzieciatkowski S, Luo Y, Lao W. Monnat RJ Jr, Emond MJ, et al. CpG island methylator phenotype is associated with response to adjuvant irinotecan-based therapy for stage III colon cancer. Gastroenterology. 2014;147(3):637-45.

10. Phipps Al, Limburg PJ, Baron JA, Burnett-Hartman AN, Weisenberger DJ, Laird PW, Sinicrope FA, Rosty C, Buchanan DD, Potter JD, et al. Association between molecular subtypes of colorectal cancer and patient survival. Gastroenterology. 2015;148(1):77-87.

11. Kang KJ, Min BH, Ryu KJ, Kim KM, Chang DK, Kim JJ, Rhee JC, Kim YH. The role of the $\mathrm{CpG}$ island methylator phenotype on survival outcome in colon cancer. Gut Liver. 2015;9(2):202-7.

12. Siena S, Sartore-Bianchi A, Di Nicolantonio F, Balfour J, Bardelli A. Biomarkers predicting clinical outcome of epidermal growth factor receptor-targeted therapy in metastatic colorectal cancer. J Natl Cancer Inst. 2009;101(19): 1308-24.

13. Toyota M, Ahuja N, Ohe-Toyota M, Herman JG, Baylin SB, Issa JP. CpG island methylator phenotype in colorectal cancer. P Natl Acad Sci USA. 1999; 96(15):8681-6.

14. Issa JP. CpG island methylator phenotype in cancer. Nat Rev Cancer. 2004; 4(12):988-93.

15. Limsui D, Vierkant RA, Tillmans LS, Wang AH, Weisenberger DJ, Laird PW, Lynch CF, Anderson KE, French AJ, Haile RW, et al. Cigarette smoking and colorectal cancer risk by molecularly defined subtypes. J Natl Cancer I. 2010; 102(14):1012-22.

16. Mack SC, Witt H, Piro RM, Gu L, Zuyderduyn S, Stutz AM, Wang X, Gallo M, Garzia L, Zayne K, et al. Epigenomic alterations define lethal CIMP-positive ependymomas of infancy. Nature. 2014;506(7489):445-50.

17. Tahara T, Yamamoto E, Madireddi P, Suzuki H, Maruyama R, Chung W, Garriga J, Jelinek J, Yamano HO, Sugai T, et al. Colorectal carcinomas with CpG island methylator phenotype 1 frequently contain mutations in chromatin regulators. Gastroenterology. 2014;146(2):530-8.

18. Shen L, Catalano PJ, Benson AB 3rd, O'Dwyer P, Hamilton SR, Issa JP. Association between DNA methylation and shortened survival in patients with advanced colorectal cancer treated with 5 -fluorouracil based chemotherapy. Clin Cancer Res. 2007;13(20):6093-8.

19. Min BH, Bae JM, Lee EJ, Yu HS, Kim YH, Chang DK, Kim HC, Park CK, Lee SH, Kim KM, et al. The CpG island methylator phenotype may confer a survival 
benefit in patients with stage II or III colorectal carcinomas receiving fluoropyrimidine-based adjuvant chemotherapy. BMC Cancer. 2011;11:344.

20. Davies H, Bignell GR, Cox C, Stephens P, Edkins S, Clegg S, Teague J, Woffendin H, Garnett MJ, Bottomley W, et al. Mutations of the BRAF gene in human cancer. Nature. 2002;417(6892):949-54.

21. Prahallad A, Sun C, Huang S, Di Nicolantonio F, Salazar R, Zecchin D, Beijersbergen RL, Bardelli A, Bernards R. Unresponsiveness of colon cancer to BRAF(V600E) inhibition through feedback activation of EGFR. Nature. 2012;483(7387):100-3.

22. Ogino S, Shima K, Meyerhardt JA, McCleary NJ, Ng K, Hollis D, Saltz LB, Mayer RJ, Schaefer $P$, Whittom $R$, et al. Predictive and prognostic roles of BRAF mutation in stage III colon cancer: results from intergroup trial CALGB 89803. Clin Cancer Res. 2012;18(3):890-900.

23. Clancy C, Burke JP, Kalady MF, Coffey JC. BRAF mutation is associated with distinct clinicopathological characteristics in colorectal cancer: a systematic review and meta-analysis. Color Dis. 2013;15(12):e711-8.

24. Carethers JM, Murali B, Yang B, Doctolero RT, Tajima A, Basa R, Smith $E J$, Lee $M$, Janke $R, N g o T$, et al. Influence of race on microsatellite instability and CD8+ T cell infiltration in colon cancer. PloS one. 2014; 9(6):e100461.

25. Li HT, LU YY, An YX, Wang X, Zhao QC. KRAS, BRAF and PIK3CA mutations in human colorectal cancer: relationship with metastatic colorectal cancer. Oncol Rep. 2011;25(6):1691-7.

26. Liou JM, Wu MS, Shun CT, Chiu HM, Chen MJ, Chen CC, Wang HP, Lin JT, Liang JT. Mutations in BRAF correlate with poor survival of colorectal cancers in Chinese population. Int J Color Dis. 2011;26(11):1387-95.

27. Farina-Sarasqueta A, van Lijnschoten G, Moerland E, Creemers GJ, Lemmens VE, Rutten HJ, van den Brule AJ. The BRAF V600E mutation is an independent prognostic factor for survival in stage II and stage III colon cancer patients. Ann Oncol. 2010;21(12):2396-402.

28. Hsieh LL, Er TK, Chen CC, Hsieh JS, Chang JG, Liu TC. Characteristics and prevalence of KRAS, BRAF, and PIK3CA mutations in colorectal cancer by high-resolution melting analysis in Taiwanese population. Clin Chim Acta. 2012;413(19-20):1605-11.

29. Ye JX, Liu Y, Qin Y, Zhong HH, Yi WN, Shi XY. KRAS and BRAF gene mutations and DNA mismatch repair status in Chinese colorectal carcinoma patients. World J Gastroenterol. 2015;21(5):1595-605.

30. Yokota T, Ura T, Shibata N, Takahari D, Shitara K, Nomura M, Kondo C, Mizota A, Utsunomiya S, Muro $\mathrm{K}$, et al. BRAF mutation is a powerful prognostic factor in advanced and recurrent colorectal cancer. $\mathrm{Br} J$ Cancer. 2011;104(5):856-62.

31. Weisenberger DJ, Siegmund KD, Campan M, Young J, Long TI, Faasse MA, Kang GH, Widschwendter M, Weener D, Buchanan D, et al. CpG island methylator phenotype underlies sporadic microsatellite instability and is tightly associated with BRAF mutation in colorectal cancer. Nat Genet. 2006; 38(7):787-93.

32. Ogino S, Kawasaki T, Kirkner GJ, Kraft P, Loda M, Fuchs CS. Evaluation of markers for $\mathrm{CpG}$ island methylator phenotype (CIMP) in colorectal cancer by a large population-based sample. J Mol Diagn. 2007:9(3):305-14.

33. Kim JH, Shin SH, Kwon HJ, Cho NY, Kang GH. Prognostic implications of CpG island hypermethylator phenotype in colorectal cancers. Virchows Arch. 2009;455(6):485-94.

34. Ogino S, Nosho K, Irahara N, Meyerhardt JA, Baba Y, Shima K, Glickman JN, Ferrone CR, Mino-Kenudson M, Tanaka N, et al. Lymphocytic reaction to colorectal cancer is associated with longer survival, independent of lymph node count, microsatellite instability, and $\mathrm{CpG}$ island methylator phenotype. Clin Cancer Res. 2009;15(20):6412-20.

35. Jia M, Gao X, Zhang Y, Hoffmeister M, Brenner $H$. Different definitions of CpG island methylator phenotype and outcomes of colorectal cancer: a systematic review. Clin Epigenet. 2016;8:25.

36. Ogino S, Cantor M, Kawasaki T, Brahmandam M, Kirkner GJ, Weisenberger DJ, Campan M, Laird PW, Loda M, Fuchs CS. CpG island methylator phenotype (CIMP) of colorectal cancer is best characterised by quantitative DNA methylation analysis and prospective cohort studies. Gut. 2006;55(7):1000-6.

37. Toyota M, Issa JP. CpG island methylator phenotypes in aging and cancer. Semin Cancer Biol. 1999;9(5):349-57.

38. Nosho K, Shima K, Irahara N, Kure S, Baba Y, Kirkner GJ, Chen L, Gokhale S, Hazra A, Spiegelman D, et al. DNMT3B expression might contribute to CpG island methylator phenotype in colorectal cancer. Clin Cancer Res. 2009; 15(11):3663-71.
39. Bae JM, Kim JH, Cho NY, Kim TY, Kang GH. Prognostic implication of the CpG island methylator phenotype in colorectal cancers depends on tumour location. Br J Cancer. 2013;109(4):1004-12.

40. Bae JM, Kim JH, Kwak Y, Lee DW, Cha Y, Wen X, Lee TH, Cho NY, Jeong SY, Park KJ, et al. Distinct clinical outcomes of two CIMP-positive colorectal cancer subtypes based on a revised CIMP classification system. Br J Cancer. 2017;116(8):1012-20

41. Rhee YY, Kim KJ, Kang GH. CpG Island Methylator phenotype-high colorectal cancers and their prognostic implications and relationships with the serrated Neoplasia pathway. Gut Liver. 2017;11(1):38-46.

42. Vedeld HM, Merok M, Jeanmougin M, Danielsen SA, Honne H, Presthus GK, Svindland A, Sjo OH, Hektoen M, Eknaes M, et al. CpG island methylator phenotype identifies high risk patients among microsatellite stable BRAF mutated colorectal cancers. Int J Cancer. 2017;141(5):967-76.

43. Vilkin A, Niv Y, Nagasaka T, Morgenstern S, Levi Z, Fireman Z, Fuerst F, Goel A, Boland CR. Microsatellite instability, MLH1 promoter methylation, and BRAF mutation analysis in sporadic colorectal cancers of different ethnic groups in Israel. Cancer. 2009;115(4):760-9.

44. Gao XH, Yu GY, Gong HF, Liu LJ, Xu Y, Hao LQ, Liu P, Liu ZH, Bai CG, Zhang W. Differences of protein expression profiles, KRAS and BRAF mutation, and prognosis in right-sided colon, left-sided colon and rectal cancer. Sci Rep. 2017;7(1):7882.

45. English DR, Young JP, Simpson JA, Jenkins MA, Southey MC, Walsh MD, Buchanan DD, Barker MA, Haydon AM, Royce SG, et al. Ethnicity and risk for colorectal cancers showing somatic BRAF V600E mutation or CpG island methylator phenotype. Cancer Epidemiol Biomark Prev. 2008;17(7):1774-80.

46. Rozek LS, Herron CM, Greenson JK, Moreno V, Capella G, Rennert G, Gruber SB. Smoking, gender, and ethnicity predict somatic BRAF mutations in colorectal cancer. Cancer Epidemiol Biomark Prev. 2010;19(3):838-43.

47. Wang Y, Long Y, Xu Y, Guan Z, Lian P, Peng J, Cai S, Cai G. Prognostic and predictive value of $C p G$ island methylator phenotype in patients with locally advanced nonmetastatic sporadic colorectal cancer. Gastroenterol Res Pract. 2014:2014:436985.

48. Weisenberger DJ, Levine AJ, Long TI, Buchanan DD, Walters R, Clendenning $M$, Rosty $C$, Joshi AD, Stern MC, LeMarchand L, et al. Association of the colorectal CpG island methylator phenotype with molecular features, risk factors, and family history. Cancer Epidemiol Biomark Prev. 2015;24(3):512-9.

49. Kambara T, Simms LA, Whitehall VL, Spring KJ, Wynter CV, Walsh MD, Barker MA, Arnold S, McGivern A, Matsubara N, et al. BRAF mutation is associated with DNA methylation in serrated polyps and cancers of the colorectum. Gut. 2004:53(8):1137-44.

50. Bettington ML, Walker NI, Rosty C, Brown IS, Clouston AD, McKeone DM, Pearson SA, Klein K, Leggett BA, Whitehall VL. A clinicopathological and molecular analysis of 200 traditional serrated adenomas. Mod Pathol. 2015; 28(3):414-27.

\section{Publisher's Note}

Springer Nature remains neutral with regard to jurisdictional claims in published maps and institutional affiliations.
Ready to submit your research? Choose BMC and benefit from:

- fast, convenient online submission

- thorough peer review by experienced researchers in your field

- rapid publication on acceptance

- support for research data, including large and complex data types

- gold Open Access which fosters wider collaboration and increased citations

- maximum visibility for your research: over $100 \mathrm{M}$ website views per year

At $\mathrm{BMC}$, research is always in progress.

Learn more biomedcentral.com/submission 